\title{
Solid-state NMR reveals structural and dynamical properties of a membrane-anchored electron-carrier protein, cytochrome $b_{5}$
}

\author{
Ulrich H. N. Dürra ${ }^{a}$, Kazutoshi Yamamoto ${ }^{a}$, Sang-Choul Imb ${ }^{b}$, Lucy Waskell ${ }^{b}$, and Ayyalusamy \\ Ramamoorthy $\mathrm{a}^{*}$ \\ aBiophysics Research Division and Department of Chemistry, University of Michigan, Ann Arbor, MI \\ 48109-1055 \\ bDepartment of Anesthesiology, University of Michigan, and VA Medical Center, Ann Arbor, MI 48105
}

\section{Abstract}

\begin{abstract}
Cytochrome $b_{5}$ (cyt b5) is a membrane-anchored electron-carrier protein containing a heme (shown in green) in its soluble domain. It enhances the enzymatic turnover of selected members of the cytochrome P450 superfamiliy of catabolic enzymes, localized in the endoplasmic reticulum of liver cells. Remarkably, its $\alpha$-helical membrane-anchoring domain is indispensable for the cyt b5/cyt P450 interaction. Here, we present first solid-state NMR studies on holo-cyt b5 in a membrane environment, namely macroscopically oriented DMPC:DHPC bicelles. We have presented approaches to selectively investigate different domains of the protein using spectral editing NMR techniques that utilize the unique motional properties of each domain. Two-dimensional ${ }^{1} \mathrm{H}^{15} \mathrm{~N}$ HIMSELF-spectra showed PISA-wheel patterns reporting on the structure and dynamics of the membrane anchor of the protein.
\end{abstract}

The vast majority of pharmaceutical compounds in use today are hydrophobic compounds. The human metabolism oxidizes them to water soluble, excretable compounds by a superfamily of enzymes known as the cytochromes $\mathrm{P} 450$ (cyt P450). ${ }^{1}$ These enzymes are sometimes referred to as 'Mother Nature's blowtorch' because they are such strong and versatile oxidizing agents. Cytochrome $b_{5}$ (cyt b5) is a membrane bound protein which enhances the efficiency of the metabolism of selected drugs by cyt P450, by donating an electron to cyt P450.2,3 Besides augmenting drug metabolism, cyt b5 is also essential for the biosynthesis of testosterone and numerous unsaturated lipids which are necessary for maintaining the integrity of cellular membranes. ${ }^{3}$ In order to understand the molecular mechanism by which cyt b5 increases the efficiency of oxidation by cyt $\mathrm{P} 450$ in vivo, one must understand the structural folding of cyt b5 that enables it to interact with cyt P450.

Full-length rabbit cyt b5 is a $16.7 \mathrm{kDa}$ protein consisting of 3 domains: a 95 amino acid aminoterminal heme-containing domain, a 25 amino acid carboxyl-terminal membrane-anchor domain, and a 14 amino acid linker region which connects the former two. ${ }^{3,4}$ Figure $1 \mathrm{~A}$ shows a model constructed from the solution NMR structure of the soluble domain of rabbit cyt $\mathrm{b} 5 ;{ }^{5}$ the full amino acid sequence is given as supporting information. Because of the difficulties in preparing suitable samples of full-length cyt b5, X-ray ${ }^{6}$ and solution $\mathrm{NMR}^{5,7}$ studies reported the structure of the water-soluble domain of cyt b5 that lacks the transmembrane domain. Remarkably, the soluble domain alone is not able to fulfill its physiological functions.

Email: ramamoor@umich.edu.

Supporting Information Available: Sequence of cyt b5, protein expression protocol, bicelle preparation protocol, and ${ }^{31} \mathrm{P}$ spectra of bicelles, HIMSELF and PISEMA spectra, and best-fit simulations. 
3,8 Hence, it is vital to investigate the role of the membrane-bound domain and the linker region in the function of cyt b5 in order to fully understand the interaction with cyt P450. A determination of the three-dimensional structure, dynamics and the relative orientation of these domains in a membrane environment will shed light on how cyt b5 carries out its function. Here, we report the first solid-state NMR investigation of the membrane topology and related dynamic properties of full-length rabbit cyt b5 in a membrane environment.

Lipid bicelles ${ }^{9}$ were prepared from dimyristoylphosphatidylcholine (DMPC) and dihexanoylphosphatidylcholine (DHPC) in 3.5:1 molar ratio; cyt b5 was added in different amounts as described in the supporting information. The bicelle samples were magnetically aligned with the bilayer normal perpendicular to the external magnetic field for solid-state NMR experiments. The quality and extent of magnetic alignment of the bicelles containing various concentrations of cyt $\mathrm{b} 5$ were examined using ${ }^{31} \mathrm{P}$ chemical shift spectra of samples (representative spectra are given as supporting information). Bicelles containing high concentrations ( $>0.5$ mole \%) of protein did not align well, while they did align well upon lowering the protein concentration and are suitable for solid-state NMR studies. At the end of the experiments, the ${ }^{15} \mathrm{~N}-\mathrm{NMR}$ spectra and the molecular weight of cyt b5 were checked to rule out protein degradation.

${ }^{15} \mathrm{~N}$ chemical shift spectra of uniformly ${ }^{15} \mathrm{~N}$-labeled cyt b5 in aligned DMPC:DHPC bicelles show a strong dependence of the spectral lineshape on experimental conditions (Figure 1). The ${ }^{15} \mathrm{~N}$ cross-polarization (CP) spectrum obtained at $0.8 \mathrm{~ms}$ contact time (Figure 1D) displays the strongest overall intensity and most likely contains signal from the entire protein. It may be expected that the transmembrane (TM) domain of Cyt-b5 is relatively more rigid on the NMR time scale, while the water-soluble domain is less rigid. Therefore, it should be possible to distinguish resonances from these two regions based on the difference in ${ }^{15} \mathrm{~N}-{ }^{1} \mathrm{H}$ dipolar coupling values. Indeed, we have obtained markedly different ${ }^{15} \mathrm{~N}$ spectra when the CP contact time was varied to control the extent of magnetization transfer from ${ }^{1} \mathrm{H}$ to ${ }^{15} \mathrm{~N}$ nuclei (Figure $1 \mathrm{C}, \mathrm{D}, \mathrm{E})$. The spectra suggest that a shorter contact time of $0.1 \mathrm{~ms}$ is sufficient to transfer magnetization from ${ }^{1} \mathrm{H}$ to ${ }^{15} \mathrm{~N}$ in the rigid residues as found in the TM domain. These experimental conditions result in resonances in the 40 to $90 \mathrm{ppm}$ range (Figure 1E). Interestingly, the signal in the region 100 to $130 \mathrm{ppm}$ is suppressed to a great extent. On the other hand, since a longer contact time transfers magnetization to all parts of the molecule due to ${ }^{1} \mathrm{H}$ spin diffusion, the spectra at $3 \mathrm{~ms}$ (Fig. 1C) and $0.8 \mathrm{~ms}$ (Fig. 1D) contact time contain peaks in the entire ${ }^{15} \mathrm{~N}$ chemical shift frequency range. Hence, we presume that the signal appearing between 100 and $130 \mathrm{ppm}$ originates from the less-rigid residues in the soluble domain. This supposition is confirmed by a RINEPT (refocused insensitive nuclei enhanced by polarization transfer) experiment ${ }^{10}$ shown in Figure 1B. Since no decoupling pulses were employed during the evolution and refocusing delays of the RINEPT sequence, the ${ }^{15} \mathrm{~N}$ transverse magnetization evolves under the motionally averaged ${ }^{1} \mathrm{H}-{ }^{15} \mathrm{~N}$ and ${ }^{1} \mathrm{H}-{ }^{1} \mathrm{H}$ dipolar and ${ }^{1} \mathrm{H}_{-}{ }^{15} \mathrm{~N}$ scalar couplings. The time delays in the RINEPT sequence were experimentally optimized to obtain the signal from the less-rigid residues found in the soluble domain of the protein. Interestingly, the RINEPT sequence significantly suppressed the peaks from the TM domain that resonate between 40 and $90 \mathrm{ppm}$. This is mainly because the transverse magnetization from the TM region dephases relatively quickly in the evolution and refocusing periods of the RINEPT sequence due to relatively strong ${ }^{1} \mathrm{H}-{ }^{15} \mathrm{~N}$ and ${ }^{1} \mathrm{H}-{ }^{1} \mathrm{H}$ dipolar couplings.

Amide-protons can be exchanged with deuterons when exposed to $\mathrm{D}_{2} \mathrm{O}$, thus suppressing the ${ }^{15} \mathrm{~N}$ signal from water-exposed protein domains. ${ }^{11}$ This approach was utilized to suppress amide- ${ }^{15} \mathrm{~N}$ signals from the cyt $\mathrm{b} 5{ }^{15} \mathrm{~N}$-spectrum. The ${ }^{15} \mathrm{~N}$ CP-spectrum of bicelles, where water was exchanged with $\mathrm{D}_{2} \mathrm{O}$, is given in Fig. $1 \mathrm{~F}$. It contains peaks from the TM region (40 to $90 \mathrm{ppm}$ ) but not from the soluble domain (100 to $130 \mathrm{ppm}$ ) of the protein, again supporting our assignment. These 1D spectral editing approaches demonstrate that the dynamics of the 
transmembrane and soluble domains are very different and that it is possible to differentiate between the resonances from these domains, which is highly valuable to interpret data from PISEMA $^{12}$ (polarization inversion and spin exchange at the magic angle)-type experiments in terms of structure and geometry of cyt b5.

Two-dimensional separated-local-field spectra, which correlate ${ }^{15} \mathrm{~N}$ chemical shift with ${ }^{1} \mathrm{H}_{-}{ }^{15} \mathrm{~N}$ dipolar coupling, were recorded on well-oriented bicelles and are given Fig. 2. A HIMSELF $^{13}$ (heteronuclear isotropic mixing leading to spin exchange via the local field) sequence based on the PIWIMz (polarization inversion by windowless isotropic mixing) pulse scheme was used which we recently found to give better resolution when compared to the more commonly employed PISEMA sequence. ${ }^{11}$ Figure $2 \mathrm{~A}$ shows that the $1 \mathrm{D}$ spectral region assigned to the TM anchor region of the protein assumes a distinct circular PISA (polarity index slant angle) wheel pattern ${ }^{14}$, when expanded into the second dimension of ${ }^{1} \mathrm{H}^{-}{ }^{1} \mathrm{~N}$ dipolar coupling, indicative of $\alpha$-helical conformation. Spectrum $2 \mathrm{~A}$ also proves that the signal from the soluble domain, although resonating in the 100-130 ppm region characteristic for isotropic ${ }^{15} \mathrm{~N}$ chemical shifts, shows clearly resolved residual ${ }^{1} \mathrm{H}_{-}{ }^{15} \mathrm{~N}$ dipolar couplings. This suggests that the soluble domain is weakly aligned. When performed in combination with the above-mentioned spectral editing techniques, the 2D HIMSELF experiments demonstrate that it is possible to obtain the resonances from the transmembrane helical region alone by suppressing most other resonances from cyt b5 (Fig. 2B,C).

Spectral simulations were carried out to infer the tilt of the TM $\alpha$-helix from the observed PISA wheel. The dispersion of resonances in the PISA wheel is consistent with a $15^{\circ}\left( \pm 3^{\circ}\right)$ tilt of the TM helix relative to the bilayer normal. However, no single PISA wheel could give a satisfactory fit to all resonances present in the circular pattern, indicating that the helical TM domain may not be a perfect $\alpha$-helix. The Pro- 116 residue located in the middle of the TM region could induce a kink in the helical structure, which may be responsible for this observation of an imperfect PISA wheel. Further experimental studies are in progress to completely assign the spectrum.

In this study, we have presented a first structural study on holocyt b5 in a membrane environment. We found an a-helical structure for the rigid transmembrane region, with untypical geometrical imperfection due to the Pro residue, and very fast dynamics for the soluble domain. We believe that the presented results point out a versatile way to investigate numerous structural and dynamic properties of cyt $\mathrm{b} 5$ and its physiologically important interaction with cyt P450. The successful demonstration of spectral editing approaches will significantly reduce the difficulties in the resonance assignment of cyt b5. Moreover, the combination of these unique NMR spectral editing methods will be applicable in the structural and dynamical studies of a large number of other membrane proteins as well.

\section{Supplementary Material}

Refer to Web version on PubMed Central for supplementary material.

\section{Acknowledgment}

This study was supported by research funds from NIH (AI054515 to A.R and GM035533 to L.W.) and a VA Merit Review grant to L. W.

\section{References}

1. Denisov IG, Makris TM, Sligar SG, Schlichting I. Chem. Rev 2005;105:2253. [PubMed: 15941214] Guengerich, FP. Mechanism and Biochemistry. 3rd edition. de Montellano, PO., editor. Plenum Publishers; New York: 2005. 
2. Zhang H, Myshkin E, Waskell L. Biochem. Biophys. Res. Commun 2005;338:499. [PubMed: 16182240]

3. Vergères G, Waskell L. Biochimie 1995;77:604. [PubMed: 8589071]Schenkman JB, Jansson I. Pharmacol. Ther 2003;97:139. [PubMed: 12559387]

4. Mulrooney SB, Waskell L. Protein Expr. Purif 2000;19:173. [PubMed: 10833404]

5. Banci L, Bertini I, Rosato A, Scacchieri S. Eur. J. Biochem 2000;267:755. [PubMed: 10651812]

6. Durley RCE, Mathews FS. Acta Cryst 1996;D52:65.

7. Guiles RD, Basus VJ, Kuntz ID, Waskell L. Biochemistry 1992;31:11365. [PubMed: 1332756]Muskett FW, Kelly GP, Whitford D. J. Mol. Biol 1996;258:172. [PubMed: 8613986]

8. Clarke TA, Im S-C, Bidwai A, Waskell L. J. Biol. Chem 2004;279:36809. [PubMed: 15194706]

9. Sanders CR, Prestegard JH. Biophys. J 1990;58:447. [PubMed: 2207249]Sanders CR, Sanders CR, Hare BJ, Howard KP, Prestegard JH. Prog. Nucl. Magn. Reson. Spectrosc 1994;26:421.Prosser RS, Evanics F, Kitevski JL, Al-Abdul-Wahid MS. Biochemistry 2006;45:8453. [PubMed: 16834319]Lu JX, Damodaran K, Lorigan GA. J. Magn. Reson 2006;178:283-287. [PubMed: 16275029]

10. Burum DP, Ernst RR. J. Magn. Reson 1980;39:163.

11. Li R, Woodward C. Protein Sci 1999;8:1571. [PubMed: 10452602]

12. Wu CH, Ramamoorthy A, Opella SJ. J. Magn. Reson. Ser. A 1994;109:270.Ramamoorthy A, Wei Y, Lee DK. Ann. Rep. NMR Spectrosc 2004;52:1-52. Yamamoto K, Lee DK, Ramamoorthy A. Chem. Phys. Lett 2005;407:289-293.

13. Dvinskikh SV, Yamamoto K, Ramamoorthy A. J. Chem. Phys 2006;125:34507. [PubMed: 16863362] Dvinskikh S, Dürr U, Yamamoto K, Ramamoorthy A. J. Am. Chem. Soc 2006;128:6326-6327. [PubMed: 16683791]

14. Marassi FM, Opella SJ. J. Magn. Reson 2000;144:150. [PubMed: 10783285]Denny JK, Wang, et al. J. Magn. Reson 2000;144:217. [PubMed: 10828190] 

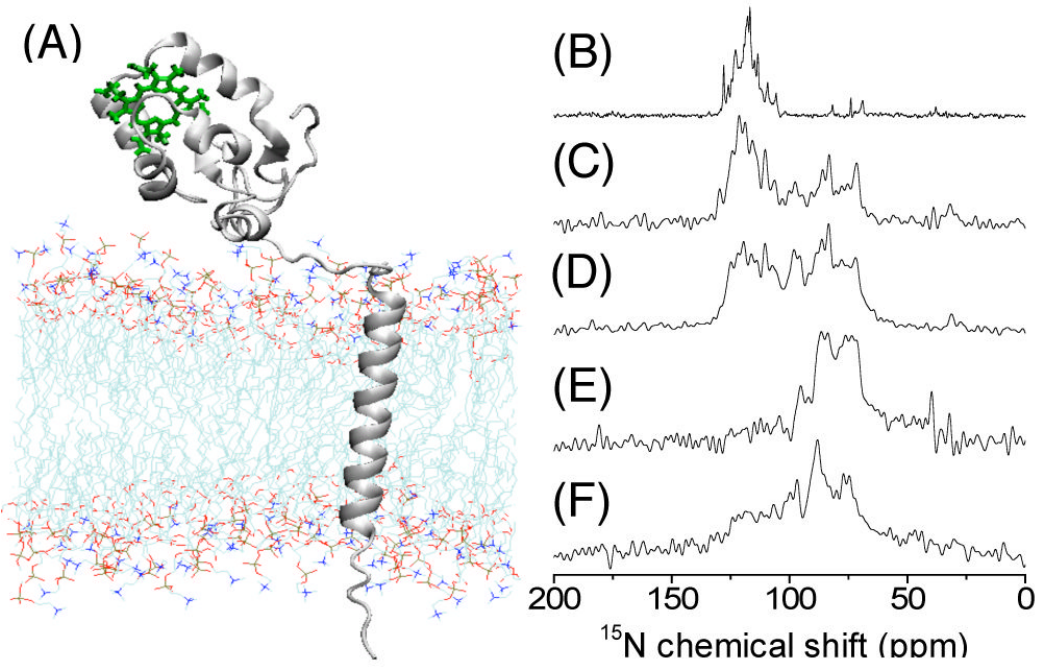

Figure 1.

Molecular model and ${ }^{15} \mathrm{~N}-\mathrm{NMR}$ spectra of uniformly aligned DMPC:DHPC bicelles containing ${ }^{15} \mathrm{~N}$-labeled cyt b5. The model (A) shows cyt b5 in the context of a DMPC bilayer; the transmembrane, linker, and heme-carrying soluble domains are evident. The ${ }^{15} \mathrm{~N}-\mathrm{RINEPT}$ spectrum (B) shows spectral intensity only in the 100-130 ppm region, consistent with a high mobility of the soluble domain. In the RINEPT sequence, 2.6 and $1.3 \mathrm{~ms}$ were used in the first (before the pair of $90^{\circ}$ pulses) and second (after the pair of $90^{\circ}$ pulses) delays, respectively. This spectral region (100-130 ppm) also shows peaks in cross-polarization (CP) spectra at 3.0 $\mathrm{ms}(\mathrm{C})$ and $0.8 \mathrm{~ms}$ (D) contact times. At a short contact time of $0.1 \mathrm{~ms}(\mathrm{E})$, however, spectral intensity is mostly observed in the range from 40 to $90 \mathrm{ppm}$. This spectral component is most likely from the relatively immobile transmembrane domain. $\mathrm{A}_{2} \mathrm{O}$-exchange experiment $(\mathrm{F})$ with a $0.8 \mathrm{~ms}$ contact time confirms this spectral assignment. 


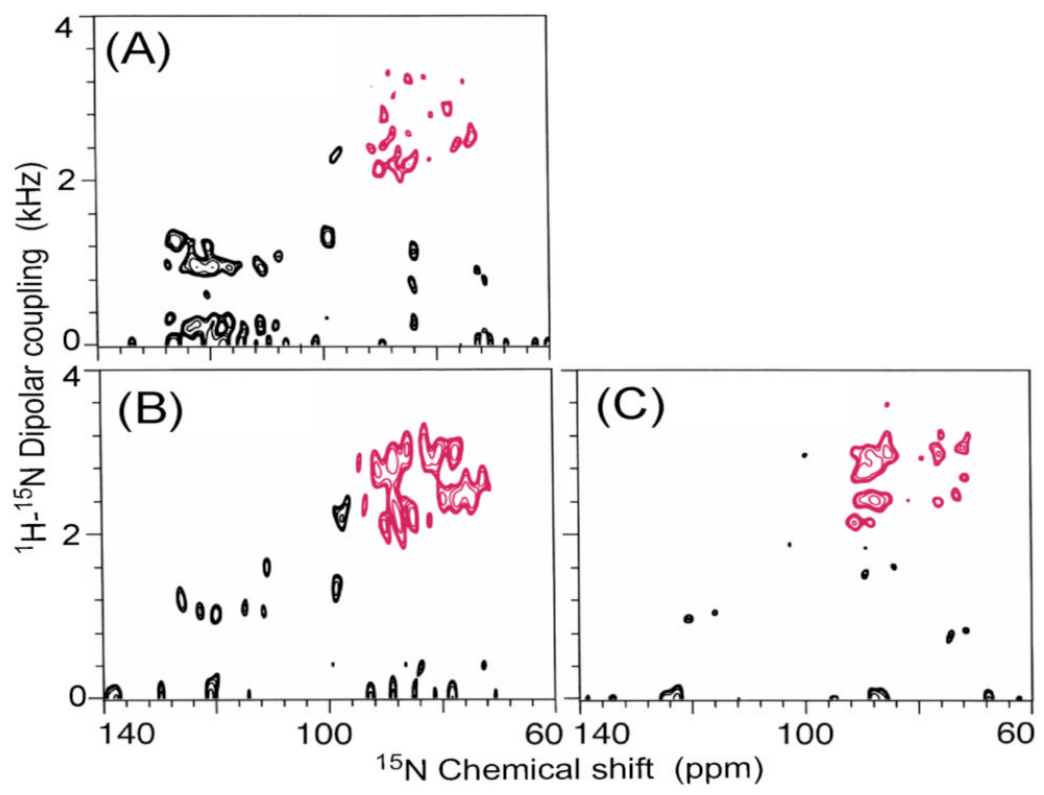

Figure 2.

${ }^{15} \mathrm{~N}$-HIMSELF spectra of U- ${ }^{15} \mathrm{~N}$-cyt b5, recorded with a CP contact time of $0.8 \mathrm{~ms}(\mathrm{~A})$ and $0.1 \mathrm{~ms}(\mathrm{~B})$. (C) Recorded after the water in the bicelle sample was replaced by $\mathrm{D}_{2} \mathrm{O}$, suppressing the spectral contributions of the soluble domain. 